\title{
Resiliencia y factores sociodemográficos involucrados en la presencia de sintomatología depresiva, ansiosa y de estrés en población adulta chilena durante la pandemia de COVID-19
}

\author{
Patricio Riquelme-Lobos y Paola Raipán-Gómez \\ Centro Psicosocial Trafkin, IX Región De La Araucanía, Chile
}

Resilience and sociodemographic factors involved in the presence of depressive, anxiety and of stress symptoms in the Chilean adult population during the COVID-19 pandemic

\begin{abstract}
The COVID-19 pandemic has caused a great impact on people's mental health. The objective of this study was to examine the role of resilience and sociodemographic factors involved in the presence of depressive, anxiety and stress symptoms in the Chilean adult population during the COVID-19 pandemic. A sample of 501 participants answered the Depression, Anxiety and Stress Scales (DASS-21) and the 10-item Connor-Davidson Resilience Scale (CD-RISC-10). The results indicate that there are high prevalence rates of mental health symptoms during the current health crisis. We found that resilience is inversely associated with the three types of symptoms, also turning out to be a predictor variable for them. It may be concluded that there are groups with a higher risk for experiencing higher levels of depressive, anxiety and stress symptomatology, which indicates the importance of developing strategies that promote mental health.
\end{abstract}

Keywords: COVID-19; resilience; depression; anxiety; stress.

Resumen: La pandemia COVID-19 ha provocado un gran impacto en la salud mental de las personas. El objetivo del estudio fue examinar el rol de la resiliencia y de factores sociodemográficos involucrados en la presencia de sintomatología depresiva, ansiosa y de estrés en población adulta chilena durante la pandemia por COVID-19. Una muestra de 501 participantes respondió las Escalas de Depresión, Ansiedad y Estrés (DASS-21) y la Escala de Resiliencia de Connor-Davidson de 10 ítems (CD-RISC-10). Los resultados indican que existen altas tasas de prevalencia de sintomatología de salud mental durante la actual crisis sanitaria. Encontramos que la resiliencia se asocia de forma inversa a los tres tipos de sintomatología, resultando ser, además, una variable predictora para estos. Se concluye que existen grupos con mayor riesgo de experimentar niveles más altos de sintomatología depresiva, ansiosa y de estrés, lo que indica la importancia de desarrollar estrategias que promuevan la salud mental.

Palabras clave: COVID-19; resiliencia; depresión; ansiedad; estrés.

\section{Introducción}

A finales de diciembre de 2019, la Organización Mundial de la Salud (OMS, 2020) fue notificada por primera vez sobre el actual brote de enfermedad por coro- navirus (COVID-19), detectado en Wuhan, China. El 11 de marzo de 2020 la OMS declaró pandemia mundial de esta enfermedad dada la alta propagación del virus (OMS, 2020a). Desde la declaración de pandemia por COVID-19 por parte de la OMS, distintos países han

Recibido: 17 de julio 2020; aceptado: 27 de marzo 2021

Correspondencia: Patricio Riquelme-Lobos, Centro Psicosocial Trafkin, Avenida José Manuel Balmaceda, n. ${ }^{\circ}$ 246, Loncoche, 4970000 IX Región De La Araucanía, Chile. Correo-e: priquelmelobos@gmail.com 
planificado y realizado estrategias para prevenir el número de contagios de su población, entre estas se encuentran la restricción de libertades individuales con aislamientos impuestos y toques de queda (Anguita y Dagnino, 2020). En el caso particular de Chile, la pandemia ha provocado adaptaciones y desafíos sociales y políticos, entre ellos el cierre de las fronteras aéreas internacionales y nacionales, declaración del estado de excepción constitucional por catástrofe o calamidad pública a partir del 18 de marzo de 2020 y la promulgación de cuarentenas obligatorias en distintas regiones del país (Ministerio del Interior y Seguridad Pública, 2020).

Si bien el aislamiento social promulgado por el estado de Chile y otros países afectados, tendrá como fin reducir el contagio, el distanciamiento social, incrementaría la posibilidad de desarrollar problemas mentales o psicológicos (Huarcaya-Victoria, 2020). De igual forma la falta de comunicación interpersonal podría empeorar el desarrollo de trastornos depresivos o ansiosos (Xiao, 2020). Igualmente el carácter incontrolable de la pandemia, la falta de información de la enfermedad y su condición de virus impredecible podrían generar alteraciones psicológicas (Sandín et al., 2020).

A lo largo de los años la población chilena se ha enfrentado con relativa frecuencia a distintos tipos de crisis, entre ellas desastres naturales como terremotos, aluviones o erupciones de volcanes (Anguita y Dagnino, 2020). Este tipo de desastres suele dejar consecuencias tanto físicas como psicológicas y dependerá de factores como la edad o el género, el grado de impacto que tendrán estos en la salud mental. En este sentido se ha establecido en la literatura a las mujeres, niños, adolescentes, ancianos y personas de escasos recursos como población de mayor riesgo (Groome y Soureti, 2004; Karet al., 2007; Ticehurst et al., 1996; Tolin y Foa, 2006). Para el caso de las consecuencias psicológicas en desastres naturales o eventos traumáticos, la literatura reporta el desarrollo de trastornos de estrés post-traumáticos, sintomatología depresiva o ansiosa (Díaz et al., 2011; Figueroa y Cortés, 2016; Cova y Rincón, 2010).

Hasta la fecha (junio de 2020) en Chile ha transcurrido un periodo de tiempo de 3 meses desde que se declaró el estado de excepción, lo que significa que la población ha experimentado cambios en su rutina diaria tales como aislamiento social, cuarentenas, cordones sanitarios, aduanas sanitarias, restricción de libertad de locomoción y reunión, entre otras, con el fin de mitigar y controlar la propagación de contagios por COVID-19. Por otro lado, según el reporte del Ministerio de Salud del 24 de junio de 2020, las cifras de casos confirmados acumulados de coronavirus a nivel nacional ascienden a un total de 254.416 y un total de 4.731 fallecidos. Todas las situa- ciones mencionadas anteriormente influyen sin duda en la salud mental de las personas, siendo muy probable que el impacto psicológico producto de la actual pandemia esté condicionado por estos factores.

La situación actual producto de la pandemia se asocia con importantes efectos psicológicos negativos para las personas (Brooks et al., 2020), tales como síntomas depresivos, de estrés, ansiosos, de confusión o de ira, etc. (Brooks et al., 2020; Sandín et al., 2020, 2021; Wang et al., 2020), y específicamente en Chile, se ha visto un incremento de servicios psicológicos, lo que da cuenta de un aumento de la preocupación de las posibles consecuencias de salud mental que podría tener esta pandemia en la población (Anguita y Dagnino, 2020), por lo que resulta relevante considerar y dimensionar las consecuencias en la salud mental de la población debido al aislamiento social como medida preventiva.

Respecto a la sintomatología depresiva, ansiosa y de estrés en Chile, escasas son las investigaciones durante el panorama actual, pero los estudios existentes se han centrado en estudiar esta sintomatología de salud mental como estados de ánimo recurrentes durante la crisis sanitaria producto del COVID-19 (Anguita y Dagnino, 2020; Asociación de Municipalidades de Chile [AMUCH], 2020; Ipsos, 2020).

Al revisar la evidencia científica disponible sobre sintomatología depresiva, ansiosa y de estrés, ésta señala altas tasas de prevalencia en estos tres cuadros sintomatológicos durante la actual pandemia. En un estudio realizado en población general china durante la fase inicial del brote de COVID-19, se reportó que el 16.5\% de la muestra presentaba síntomas depresivos en un nivel moderado a severo (Wang et al., 2020). Asimismo, un estudio con población salvadoreña durante cuarentena domiciliaria producto de la pandemia, señala que el $74.9 \%$ de la muestra manifestó síntomas leves de depresión y un $13.3 \%, 2.7 \%$ y un $9.1 \%$ presentó síntomas moderados, severos y extremadamente severos, respectivamente (Orellana y Orellana, 2020). En el contexto chileno, un estudio realizado con una muestra de 600 participantes, reveló que el $29 \%$ de los sujetos presentó un nivel alto de estado de ánimo de depresión (Ipsos, 2020).

En cuanto a la sintomatología ansiosa, un estudio con población general del Reino Unido durante la pandemia, indicó que el $21.63 \%$ de los participantes presentó sintomatología ansiosa (Shevlin et al., 2020). Así también, un estudio con población boliviana presentó cifras similares, ya que el $24 \%$ manifestó síntomas de ansiedad (Wanderley et al., 2020). Por su parte, en Chile un estudio realizado con una muestra de 663 participantes, indicó que el $56 \%$ reportó sentimientos de ansiedad que han aparecido producto de la crisis sanitaria (AMUCH, 2020), mientras que en otro estudio se señala que el $60 \%$ 
de los participantes sintieron ansiedad como efecto del aislamiento social (Anguita y Dagnino, 2020).

Por último, en lo que respecta a la sintomatología de estrés, un estudio realizado con población italiana, reportó que el $72.8 \%$ de la muestra se encontraba en un rango promedio, un $14.6 \%$ en un rango alto y un $12.6 \%$ en un rango extremadamente alto en sintomatología de estrés (Mazza et al., 2020). Tanto a nivel latinoamericano como nacional, las cifras de sintomatología de estrés siguen tendencias similares, ya que en un estudio con población salvadoreña el $77.9 \%$ presentó síntomas leves de ansiedad (Orellana y Orellana, 2020), y en el caso de Chile un estudio encontró que el 66\% de los participantes presentó un nivel alto de estado de ánimo de estrés durante la actual crisis sanitaria (Ipsos, 2020).

En conclusión, podemos observar que las tasas de sintomatología tanto depresiva, ansiosa y de estrés son preocupantes, resultando relevante poder examinar aquellos factores de riesgo que pueden propiciar la aparición de estos tres cuadros. La variable género es uno de los factores más recurrentes y los estudios surgidos durante la crisis sanitaria demostraron que pertenecer al género femenino se asoció con mayores niveles de depresión, ansiedad y estrés, tanto a nivel nacional (Anguita y Dagnino, 2020; Ipsos, 2020) como internacional (Orellana y Orellana, 2020; Ozamiz-Etxebarria et al., 2020; Sandín et al., 2020; Wang et al., 2020).

Por otra parte, el estado laboral también se presenta como una preocupación constante producto del panorama actual (Anguita y Dagnino, 2020), ya que el impacto negativo que se ha producido en la economía, provoca una pérdida financiera y riesgo de desempleo, lo que acrecienta aún más las emociones negativas experimentadas por las personas (Ho et al., 2020). Bajo un panorama de normalidad, un estudio señala que los desempleados presentaron puntajes más altos de ansiedad y depresión (Acosta-Rodríguez et al., 2011). En el caso de la actual crisis sanitaria, un estudio indicó que las personas desempleadas o que trabajan en el hogar sin remuneración, tienen un $80 \%$ más riesgo de presentar depresión si se compara con aquellos que cuentan con un trabajo fijo; las personas a las cuales se les suspendió su salario, tienen $40 \%$ más riesgo de presentar depresión comparadas con las personas que continúan recibiendo su salario; y por último, las personas que fueron despedidas momentánea o definitivamente, tienen alrededor del doble de riesgo de presentar depresión y estrés (Instituto de Investigación en Ciencias del Comportamiento [IICC], 2020).

Así como hemos visto aquellos factores de riesgo para este tipo de sintomatologías, es necesario examinar factores protectores de la salud mental. Uno de ellos es la creencia en algún credo o religión la cual ha sido estudia- da como un factor protector de la salud mental (Salgado, 2014). Estudios indican que la religión, religiosidad y espiritualidad serían factores protectores en diversas áreas de la vida del ser humano, ya que disminuyen la depresión, la ansiedad y el estrés (Salgado, 2014). Asimismo, las creencias religiosas ayudan a evitar los síntomas depresivos (Mofidi et al., 2007), mientras que la religiosidad y espiritualidad, y la salud mental parecen estar positivamente asociadas (Bento et al., 2006).

Otro de los factores protectores de la salud mental es la resiliencia, la cual alude a las diferencias individuales que manifiestan las personas entre sí al estar enfrentadas a situaciones de riesgo, logrando un nivel «adecuado» o «normal» de desarrollo a pesar de estar viviendo situaciones adversas (Organización Panamericana de la Salud [OPS] y OMS, 1997). En este sentido, es necesario destacar que existen varias definiciones del concepto; sin embargo, para fines de este estudio se considera la resiliencia como la capacidad personal de protección frente a las situaciones de estrés y es antes un estado que un rasgo, por lo tanto es modificable (Connor y Davidson, 2003).

Respecto a las investigaciones disponibles en torno a la resiliencia y la salud mental a nivel general, éstas mencionan que existe una amplia evidencia centrada principalmente en variables psicológicas y patologías como el estrés, la ansiedad y la depresión (Asensio-Martínez et al., 2017). Asimismo, las personas resilientes desarrollarían habilidades que les permitirían mejorar y optimizar sus recursos personales para el mantenimiento de su salud (García del Castillo et al., 2016).Debido a que la crisis sanitaria causada por el COVID-19 es un acontecimiento reciente, no existen estudios que muestren evidencia científica sobre el efecto positivo de la resiliencia en la salud mental de la población adulta durante el periodo de aislamiento social y principalmente su efecto sobre la sintomatología depresiva, ansiosa y de estrés. Es por esto que solo se mencionarán estudios en torno a resiliencia y salud mental, pero relacionados a otros contextos.

En relación a la resiliencia y la sintomatología depresiva, los estudios indican una correlación inversa entre éstas, es decir que a mayores niveles de resiliencia disminuyen los niveles de depresión (Pidgeon et al., 2014; Seok et al., 2012; Yu et al., 2009). Asimismo, para la sintomatología ansiosa y de estrés, los estudios siguen tendencias similares, ya que muestran una asociación inversa entre la resiliencia y la sintomatología ansiosa y de estrés (Kemper et al., 2015; Pidgeon et al., 2014; Yu et al., 2009).

De acuerdo a lo anterior, podemos observar que hasta la fecha existen pocos estudios en Chile sobre el impacto en la salud mental durante la actual pandemia 
(Anguita y Dagnino, 2020; Asociación de Municipalidades de Chile [AMUCH], 2020; Ipsos, 2020), presentando algunas limitaciones. Los estudios se centran en describir las sensaciones, emociones o estados de ánimo de la población producto del aislamiento social ocasionado por el COVID-19, pero no utilizan instrumentos que determinen sintomatología de salud mental y sus grados de afectación en las personas. Además, no consideran factores protectores de la salud mental durante la actual pandemia. Por otro lado, respecto a resiliencia y salud mental durante la crisis sanitaria actual, no existen estudios aún que consideren dichas variables, ni tampoco se han estudiado en población adulta chilena específicamente.

El objetivo general del presente estudio consistió en examinar el rol de la resiliencia y de factores sociodemográficos involucrados en la presencia de sintomatología depresiva, ansiosa y de estrés en población adulta chilena durante la pandemia de COVID-19. Los objetivos específicos fueron: (1) comparar la sintomatología depresiva, ansiosa y de estrés según factores sociodemográficos como género, estado laboral y creencias, (2) analizar si existen diferencias en la resiliencia según factores sociodemográficos como género, estado laboral y creencias, y (3) determinar la correlación y/o predicción de las variables resiliencia y sintomatología depresiva, ansiosa y de estrés.

\section{Método}

\section{Participantes}

La muestra se constituyó por 501 participantes pertenecientes a 86 ciudades o localidades de Chile, seleccionados mediante un muestreo no probabilístico de bola de nieve. Los criterios de inclusión de este estudio fueron: tener 18 o más años y tener nacionalidad chilena.

El rango de edad de los participantes fue de 18 a 72 años $(M=29.19, D T=9.31)$, de los cuales el $75.2 \%$ eran mujeres y el $24.8 \%$ eran hombres. La mayoría vivía con 4 personas (incluido el participante) y pasaban 24 horas diarias (en promedio) en la casa para evitar exponerse al COVID-19.

La mayoría de los participantes eran solteros (78.2\%), con un nivel educacional universitario completo (44.1\%), seguido de un nivel universitario incompleto o superior técnico completo (30.7\%). El 53.1\% de los sujetos estaba desempleado y el $56.3 \%$ era creyente en algún credo o religión. Los participantes en su mayoría, se sentían preocupados por la pérdida de sus ingresos económicos por causa de esta crisis sanitaria $(89.8 \%)$, se sentían satisfechos por el desempeño de los profesionales del sis- tema de salud (84.4\%) y contaban con apoyo familiar durante esta crisis sanitaria (87.6\%). Las características sociodemográficas de la muestra se observan en más detalle en la Tabla 1.

Tabla 1. Características sociodemográficas de la muestra

\begin{tabular}{|c|c|c|}
\hline Variables sociodemográficas & $n$ & $\%$ \\
\hline \multicolumn{3}{|l|}{ Género } \\
\hline Masculino & 124 & 24.8 \\
\hline Femenino & 377 & 75.2 \\
\hline \multicolumn{3}{|l|}{ Estado civil } \\
\hline Soltero/a & 392 & 78.2 \\
\hline Casado/a & 81 & 16.2 \\
\hline Divorciado/a & 14 & 2.8 \\
\hline Conviviente civil (AUC) & 14 & 2.8 \\
\hline \multicolumn{3}{|l|}{ Nivel educacional } \\
\hline Básica incompleta o menos & 2 & 0.4 \\
\hline Básica completa & 1 & 0.2 \\
\hline Media incompleta o media técnicacompleta & 16 & 3.2 \\
\hline Media completa o superior técnica incompleta & 64 & 12.8 \\
\hline $\begin{array}{l}\text { Universidad incompleta o superiortécnica com- } \\
\text { pleta }\end{array}$ & 154 & 30.7 \\
\hline Universitaria completa & 221 & 44.1 \\
\hline Postgrado & 43 & 8.6 \\
\hline \multicolumn{3}{|l|}{ Estado laboral } \\
\hline Empleado & 235 & 46.9 \\
\hline Desempleado & 266 & 53.1 \\
\hline \multicolumn{3}{|l|}{ Razón del desempleo } \\
\hline Estar estudiando o capacitándose & 121 & 45.7 \\
\hline $\begin{array}{l}\text { Haber perdido el trabajo producto de la } \\
\text { crisis sanitaria }\end{array}$ & 52 & 19.6 \\
\hline Otras razones & 92 & 34.7 \\
\hline \multicolumn{3}{|l|}{ Creencia en algún credo o religión } \\
\hline Sí & 282 & 56.3 \\
\hline No & 219 & 43.7 \\
\hline \multicolumn{3}{|l|}{$\begin{array}{l}\text { Sentir que tus creencias te han permitido afron- } \\
\text { tar de mejor manera esta crisis sanitaria }\end{array}$} \\
\hline Sí & 139 & 49.8 \\
\hline No & 51 & 18.3 \\
\hline A veces & 89 & 31.9 \\
\hline \multicolumn{3}{|l|}{$\begin{array}{l}\text { Preocupación por la pérdida de tus ingresos } \\
\text { económicos por causa de esta crisis sanitaria }\end{array}$} \\
\hline Sí & 450 & 89.8 \\
\hline No & 51 & 10.2 \\
\hline \multicolumn{3}{|l|}{$\begin{array}{l}\text { Sentirse satisfecho por el desempeño de los pro- } \\
\text { fesionales del sistema de salud }\end{array}$} \\
\hline Sí & 423 & 84.4 \\
\hline No & 78 & 15.6 \\
\hline \multicolumn{3}{|l|}{ Apoyo familiar durante esta crisis sanitaria } \\
\hline Sí & 439 & 87.6 \\
\hline No & 62 & 12.4 \\
\hline
\end{tabular}




\section{Diseño y procedimiento}

Este estudio es cuantitativo, con un diseño no experimental de carácter transversal y con un alcance descriptivo-correlacional. Su objetivo es examinar el rol de la resiliencia y de factores sociodemográficos ante la presencia de sintomatología depresiva, ansiosa y de estrés en población adulta chilena durante la crisis sanitaria ocasionada por el COVID-19.

Ante el panorama actual y de acuerdo a las medidas de prevención recomendadas por el gobierno de Chile, se contactó a los participantes de forma remota, es decir fueron invitados electrónicamente por los investigadores por medio de correos electrónicos y redes sociales, donde podían acceder a un enlace que los dirigía a la encuesta en línea a través de la plataforma Google Formulario. Los datos fueron recolectados desde el 27 de abril al 24 de mayo de 2020. Todos los participantes aceptaron previamente un consentimiento informado, el cual indicaba el objetivo del estudio y el carácter voluntario, anónimo y confidencial de la participación. El periodo de tiempo destinado a la recolección de los datos coincidió con el primer brote de COVID-19 en Chile, por lo tanto la población se encontraba en confinamiento producto del estado de excepción.

\section{Instrumentos}

Cuestionario Sociodemográfico. Cuestionario construido por los investigadores para recopilar datos sociodemográficos como edad, género, nivel educacional, estado laboral y creencias en algún credo o religión.

Escalas de Depresión, Ansiedad y Estrés (DASS-21; Lovibond y Lovibond, 1995; versión chilena de Antúnez y Vinet, 2012). Se trata de una escala de auto-reporte de 21 ítems que evalúa el grado, la intensidad y la frecuencia en que se ha experimentado la sintomatología depresiva, ansiosa y de estrés, durante la última semana. La escala consta de cuatro alternativas de respuesta que varían entre 0 («No describe nada de lo que me pasó o sentí en la semana») y 3 («Sí, esto me pasó mucho, o casi siempre»). El instrumento cuenta con tres subescalas las cuales contienen siete ítems cada una y la puntuación por subescala varía entre 0 y 21 puntos, donde a mayor puntaje obtenido se estima que existe mayor presencia de sintomatología depresiva, ansiosa y de estrés. Los puntos de corte de cada subescala son, en el caso de depresión mayor que 5, para ansiedad mayor que $4 \mathrm{y}$, por último, para estrés mayor que 5 (Román, Santibáñez, y Vinet, 2016). Por otro lado, la prevalencia de sintomatología recogida en esta investigación mediante este instrumento, da cuenta que el $42.7 \%$ de la muestra total presentó puntajes superiores al punto de corte para sintomatología de- presiva, un $35.7 \%$ presentó sintomatología ansiosa y un $58.4 \%$, sintomatología de estrés, mientras que un $26.1 \%$ de los participantes presentó conjuntamente sintomatología depresiva, ansiosa y de estrés. En relación a la fiabilidad del instrumento DASS-21, se realizó el cálculo de ésta por medio del estadístico alfa de Cronbach. Para la escala total del instrumento la fiabilidad fue de .95 , mientras que, para sus dimensiones de depresión, ansiedad y estrés, ésta fue de $.89, .89$, y .88 respectivamente.

Escala de Resiliencia de Connor-Davidson (CDRISC; Connor y Davidson, 2003). Este instrumento fue adaptado por Campbell-Sills y Stein (2007) resultando una escala unidimensional de 10 ítems (CD-RISC-10). Para este estudio se utilizó la versión española del CDRISC de 10 ítems de Notario-Pacheco et al. (2011). Se trata de una escala de auto-reporte de 10 ítems que busca estimar el nivel de resiliencia de una persona según el grado de acuerdo e identificación con las frases que se les presentan. La escala consta de cinco alternativas de respuesta que varían entre 0 («Nunca») y 4 («Casi siempre»). El puntaje total del instrumento se calcula sumando las respuestas obtenidas en cada ítem, el cual varía entre 0 y 40 puntos, donde a mayor puntaje obtenido se estima un nivel más alto de resiliencia. Por otra parte, se calculó la media de la escala total del instrumento CDRISC-10, resultando que el 53.6\% de la población adulta chilena percibe un alto nivel de resiliencia, mientras que un $46.3 \%$ percibe un bajo nivel de resiliencia. En este estudio, el instrumento CD-RISC-10 presentó una adecuada consistencia global, obteniendo un alfa de Cronbach de .83 para su escala total.

\section{Análisis estadísticos}

Para comparar la sintomatología depresiva, ansiosa y de estrés según factores sociodemográficos como género, estado laboral y creencias, como indica el objetivo 1, se utilizó la prueba $t$ de Student para grupos independientes. Asimismo, para el objetivo 2 que pretendía analizar si existen diferencias en la resiliencia según factores sociodemográficos como género, estado laboral y creencias, se utilizó la prueba $t$ de Student para grupos independientes.

Por último, para determinar la correlación y/o predicción de las variables resiliencia y sintomatología depresiva, ansiosa y de estrés, se utilizó el coeficiente de correlación de Pearson para establecer asociación entre las variables resiliencia y los tres tipos de sintomatología. Finalmente, se utilizó la prueba de regresión lineal simple con el método Introducir para analizar el efecto predictivo de la resiliencia sobre la sintomatología depresiva, ansiosa y de estrés. Los análisis se realizaron con el programa estadístico SPSS.25. 


\section{Resultados}

Para responder al primer objetivo que pretendía comparar la sintomatología depresiva, ansiosa y de estrés en función de los factores sociodemográficos (género, estado laboral y creencias), comprobamos que en el caso de la variable género la prueba $t$ indicó diferencias estadísticamente significativas entre hombres y mujeres respecto a la sintomatología depresiva $(t=3.95, p<.001)$, ansiosa $(t=4.44, p<.001)$ y de estrés $(t=4.80, p<.001)$; en las tres variables las mujeres obtuvieron medias más altas que los hombres. Respecto a la variable estado laboral, constata diferencias estadísticamente significativas entre empleados y desempleados para la sintomatología depresiva $(t=-6.74, p<.001)$, ansiosa $(t=-5.66$, $p<.001)$ y de estrés $(t=-6.21, p<.001)$; en las tres variables los desempleados obtuvieron medias más altas que los empleados. Por último, para la variable creencias encontramos diferencias estadísticamente significativas entre creyentes y no creyentes de algún credo o religión respecto a sintomatología depresiva $(t=-3.84, p<.001)$ y sintomatología de estrés $(t=-2.09, p<.05)$; en ambas dimensiones los no creyentes obtuvieron medias más altas que los creyentes de algún credo o religión. A su vez, no observamos diferencias significativas entre creyentes y no creyentes de algún credo o religión respecto a sintomatología ansiosa $(t=-1.82, p=.06)$.

Respecto al segundo objetivo, examinamos si existían diferencias en la resiliencia según los factores sociodemográficos. Para la variable género encontramos diferencias estadísticamente significativas entre hombres y mujeres respecto a la resiliencia $(t=-3.19$, $p<.01)$; los hombres obtuvieron una media más alta que las mujeres. En relación a la variable estado laboral, encontramos diferencias estadísticamente significativas entre empleados y desempleados respecto a la resiliencia $(t=6.84, p<.001)$; los empleados obtuvieron una media más alta que los desempleados. Finalmente, para la va- riable creencias no encontramos diferencias significativas entre creyentes y no creyentes de algún credo o religión respecto a la resiliencia $(t=1.76, p=.07)$.

Por último, en relación al tercer objetivo buscamos determinar la correlación y/o predicción de las variables resiliencia y sintomatología depresiva, ansiosa y de estrés. Tal y como se puede observar en la Tabla 2, encontramos una correlación estadísticamente significativa, inversa y moderada, entre sintomatología depresiva y resiliencia $(r=-.52, p<.01)$. Asimismo, encontramos que existía correlación estadísticamente significativa, inversa y débil, entre sintomatología ansiosa y resiliencia $(r=-.37, p<.01)$, e inversa y moderada entre sintomatología de estrés y resiliencia $(r=-.45, p<.01)$.

Tabla 2. Correlaciones $r$ de Pearson entre las variables $(n=501)$

\begin{tabular}{lcccc}
\hline \multicolumn{1}{c}{ Variables } & 2 & 3 & 4 & 5 \\
\hline 1. Resiliencia & $-.526^{* *}$ & $-.374^{* *}$ & $-.458^{* *}$ & $.221^{* *}$ \\
2. DASS - Depresión & - & $.689^{* *}$ & $.765^{* *}$ & $-.270^{* *}$ \\
3. DASS - Ansiedad & & - & $.793^{* *}$ & $-.196^{* *}$ \\
4. DASS - Estrés & & & - & $-.209^{* *}$ \\
5. Edad & & & & - \\
\hline
\end{tabular}

Nota. DASS: Escalas de depresión, ansiedad y estrés. $* * p<.01$.

De acuerdo a las correlaciones indicadas se realizaron tres análisis de regresión lineal simple para examinar el efecto predictivo de la resiliencia sobre la sintomatología depresiva, ansiosa y de estrés. Se incluyó como variable predictora la resiliencia y como variables criterio cada una de las dimensiones de sintomatología (depresión, ansiedad y estrés). Los resultados de estos análisis se indican en la Tabla 3. Podemos apreciar que la resiliencia predice de manera significativa los tres tipos de sintomatología (depresión, ansiedad y estrés).

Tabla 3. Predicción de la sintomatología depresiva, ansiosa y de estrés a partir de la variable resiliencia. Análisis de regresión simple con método Introducir

\begin{tabular}{lcccc}
\hline Independiente & Dependiente & $R^{2}$ (ajustado) & $\beta$ & $t$ \\
\hline 1. Resiliencia & Síntomas depresivos & .276 & -.526 & $-13.828^{* * *}$ \\
1. Resiliencia & Síntomas de ansiedad & .138 & -.374 & $-9.009^{* * *}$ \\
1. Resiliencia & Síntomas de estrés & .208 & -.458 & $-11.516^{* * *}$ \\
\hline
\end{tabular}

Nota. $* * * p<.001$.

\section{Discusión}

Por medio del presente estudio se ha pretendido examinar el rol de la resiliencia y los factores sociodemo- gráficos involucrados en la presencia de sintomatología depresiva, ansiosa y de estrés en población adulta chilena durante la pandemia; más específicamente, bajo el contexto de aislamiento social decretado como medida 
de prevención en la población para evitar la propagación del COVID-19.

En relación con el primer objetivo que pretendía comparar la sintomatología depresiva, ansiosa y de estrés según los factores sociodemográficos (género, estado laboral y creencias), constatamos que existían diferencias significativas entre hombres y mujeres respecto a la sintomatología depresiva, ansiosa y de estrés, siendo las mujeres las que reportaron mayores niveles de sintomatología en estas tres variables. Resultados similares muestran algunos estudios realizados durante esta pandemia los cuales indican que pertenecer al género femenino resultó ser un factor asociado a niveles altos de depresión, ansiedad y estrés (Orellana y Orellana, 2020; Ozamiz-Etxebarria et al., 2020; Wang et al., 2020). Esto podría deberse probablemente a que el confinamiento suele incrementar la demanda de atención de niños o personas mayores, recayendo principalmente las tareas de cuidado en las mujeres (Comisión Económica para América Latina y el Caribe [CEPAL], 2020; OMS, 2020b; Wenham et al., 2020), lo cual tendría efectos sobre la salud mental de éstas. Asimismo, durante situaciones de emergencia las mujeres se ven más afectadas en su salud mental que los hombres (Lai et al., 2020; Wenham et al., 2020).

Respecto a la comparación de sintomatología de salud mental según el estado laboral, encontramos diferencias significativas entre empleados y desempleados respecto a la sintomatología depresiva, ansiosa y de estrés, siendo los desempleados quienes presentaron mayores niveles. Este resultado podría deberse a que el desempleo producto de esta crisis genera distintos estados de ánimo negativos, debido a que se ve amenazada la fuente de ingresos y la actividad económica, además de la estabilidad familiar y personal donde no solo las personas se ven enfrentadas a consecuencias económicas, sino que también se enfrentan a consecuencias en su salud mental (Garcés y Frías, 2020); por otro lado, aquellos que quedaron sin trabajo durante esta cuarentena pudieron sufrir pérdidas financieras, lo que representa un factor de riesgo para los síntomas de trastornos psicológicos y un estresor adicional durante la pandemia (Brooks et al., 2020). En el caso de aquellas personas que se encontraban desempleadas por el hecho de estar exclusivamente dedicándose a cursar estudios superiores, probablemente experimentaron niveles más altos de depresión, ansiedad y estrés, puesto que los estudiantes representaban previo a la pandemia un grupo de riesgo, donde los estudios señalaban cifras preocupantes de sintomatología de salud mental en estudiantes universitarios (Antúnez y Vinet, 2013; Baader et al., 2014; Barrera-Herrera et al., 2019), por lo que la actual crisis sanitaria podría ser un factor más que influya en el incremento de ésta, sumándose a esto también los nuevos desafíos y demandas en el proceso educativo como la adaptación a la virtualidad, lo que desencadenaría en los estudiantes una mayor vulnerabilidad a un sinfín de situaciones (Cáceres, 2020).

En cuanto a las diferencias en sintomatología de salud mental según las creencias de las personas, encontramos diferencias significativas entre creyentes y no creyentes de algún credo o religión respecto a sintomatología depresiva y sintomatología de estrés, siendo las personas no creyentes las que presentaron mayores niveles. Este resultado concuerda con lo encontrado en la literatura, ya que las creencias religiosas actuarían como factor protector ante la depresión y el estrés, y en general para la salud mental de las personas (Mofidi et al., 2007; Salgado, 2014). Ante el actual panorama producto de la pandemia, la religiosidad y espiritualidad, han resultado ser una de las estrategias que permiten modificar una situación para aliviar los efectos nocivos que ha provocado la crisis sanitaria sobre la salud psicológica, emocional y mental, es decir, cambiar el foco del pensamiento para influir en la respuesta emocional, por medio de la búsqueda de significado a través de la religiosidad y espiritualidad (Ubillos et al., 2020). Por otro lado, durante la pandemia las personas utilizarían la religión como un medio para afrontar la adversidad y la incertidumbre que provoca una crisis (Sinding, 2020), así como también para reducir la angustia emocional que provoca una situación (Lazarus y Folkman, 1984).

Resulta llamativo que no existieran diferencias significativas entre creyentes y no creyentes de algún credo o religión respecto a la sintomatología ansiosa, ya que algunos estudios han sugerido que las creencias religiosas ayudarían a regular la afectividad negativa, en este caso a reducir la ansiedad (Campos et al., 2004; Salgado, 2014). Probablemente al estar relacionada la sintomatología ansiosa con el sufrimiento de una persona por eventos negativos futuros e inciertos (Moreno, 2020), no se han detectado diferencias de esta sintomatología de salud mental entre personas creyentes y no creyentes, ya que el futuro se vería incierto desde ambos grupos, producto de la actual pandemia.

Por otra parte, mediante el segundo objetivo examinamos la posibilidad de que existieran diferencias en la resiliencia según los factores sociodemográficos. En relación con el género se constaron diferencias significativas entre hombres y mujeres respecto a la resiliencia, siendo los hombres quienes presentaron mayores niveles. Este resultado está en la línea de otras investigaciones (Lozano-Díaz et al., 2020; Vizoso, 2019). Una posible explicación es el rol social ventajoso que ocupan los hombres frente a las mujeres, lo cual incide en sus nive- 
les de resiliencia (Hu et al., 2015). En esta línea, los hombres suelen reportar mejores indicadores de confianza o percepción de sí mismos para superar adversidades (Tomyn y Weinberg, 2016). Sin embargo, se debe considerar este resultado con cautela ya que en la presente investigación el porcentaje de mujeres es superior al de hombres.

Con respecto a la relación entre la resiliencia y el estado laboral, igualmente encontramos diferencias significativas entre empleados y desempleados, siendo los empleados quienes presentaron mayores niveles. Este resultado es consistente con la evidencia encontrada en otros estudios (Lozano-Díaz et al., 2020). Moorhouse y Caltabiano (2007) reportaron que la resiliencia ayuda a afrontar la situación de desempleo en personas adultas. En consecuencia resulta relevante considerar el papel que presenta el estado laboral en la actual crisis debido a la constante preocupación que provoca éste en la sociedad (Anguita y Dagnino, 2020).

No encontramos diferencias significativas entre creyentes y no creyentes respecto a la resiliencia, es decir, vimos que tanto las personas creyentes como las no creyentes eran igualmente resilientes. Lo cual no sigue la línea de una investigación previa realizada en Lima, la cual determinó que aquellos participantes creyentes presentaron mayores niveles de resiliencia (López et al., 2016). El resultado de este estudio podría explicarse por la forma en la que se midió la variable creencia en la muestra ya que no se indagó en su espiritualidad o religiosidad, tan solo se solicitó el reporte subjetivo de su credo.

Finalmente, respecto al tercer objetivo, el cual buscaba determinar la asociación entre la resiliencia y la sintomatología, se encontró que la resiliencia correlacionaba de forma significativa e inversa con la sintomatología depresiva, ansiosa y de estrés. Estos resultados son similares a algunos estudios que han sugerido una relación inversa entre la resiliencia y los síntomas depresivos, ansiosos y de estrés (Catalano et al., 2011; Kemper et al., 2015; Pidgeon et al., 2014; Yu et al., 2009).

Por otro lado, los análisis de regresión múltiple demostraron un efecto predictivo de la resiliencia sobre los síntomas de depresión, ansiedad y estrés, sugiriéndose un posible rol protector de esta sobre la sintomatología emocional y estrés. Sin duda otras variables protectoras de la salud mental podrían contribuir a incrementar la varianza explicada en sintomatología y estrés, tales como el apoyo social o la satisfacción con la vida, sobre las cuales se ha visto que son predictoras de una óptima salud mental (Barrera-Herrera et al., 2019; García-Cid et al., 2017). En este sentido, otros estudios también han demostrado el papel protector de la resiliencia frente al desarrollo de este tipo de sintomatología (García-Cid et al., 2017; Seok et al., 2012). Nuestros resultados están en línea con las propuestas de algunos organismos internacionales que han propuesto fomentar las políticas y programas de potenciación de la resiliencia en las poblaciones como una forma de adaptarse de mejor manera a los ciclos de crisis y lograr un mejor desarrollo de las comunidades más vulnerables (Korc et al., 2016; United Nations Children's Fund [UNICEF], 2019). Asimismo, ante el panorama actual se hace aún más relevante estudiar el papel de la resiliencia como posible factor protector, considerando el impacto psicológico y emocional asociado a la pandemia, siendo importante promover su desarrollo, como componente relevante para una adecuada adaptación psicosocial y salud mental (Cabanyes, 2010).

Resulta destacable que más del $30 \%$ de la muestra presentara niveles de riesgo en cada una de las variables de sintomatología evaluadas por las escalas DASS-21. Si comparamos este resultado con los de otros estudios que se han realizado durante la pandemia sobre el impacto en la salud mental, la prevalencia obtenida en este estudio supera a la de otras investigaciones que igualmente utilizaron el instrumentos DASS-21 con población adulta, partiendo de los niveles de sintomatología moderado a severo (Mazza et al., 2020; Orellana y Orellana, 2020; Ozamiz-Etxebarria et al., 2020; Wang et al., 2020). Esto podría explicarse porque algunos de estos estudios se realizaron en una etapa inicial del brote de COVID-19, por lo que el impacto psicológico pudo haber sido menor que en etapas posteriores.

En relación a las limitaciones presentes en este estudio, en primer lugar no es posible generalizar los resultados a toda la población adulta chilena, ya que se utilizó un muestreo no probabilístico. En segundo lugar, el porcentaje de mujeres de esta muestra era mayor que el de hombres, por lo que en futuras investigaciones podrían balancearse los porcentajes de ambos géneros. En tercer lugar, al utilizar una encuesta en línea solo se llegó a aquellos participantes que contaban con acceso a internet. Por último, este estudio solo consideró algunos factores que influyen en la salud mental de las personas, dejando fuera otras variables psicológicas relevantes, como por ejemplo, mecanismos de afrontamiento, relaciones personales, variables de personalidad, tiempo en familia, entre otras, por lo que sería importante considerar en futuros estudios una mayor variabilidad de factores, tanto positivos como negativos, y así poder explicar más ampliamente el efecto de éstos sobre la salud mental durante la actual crisis sanitaria.

Para futuras investigaciones se podrían incluir distintos tipos de muestras y así llegar a una mayor cantidad de 
población con otras características. De igual forma, sería importante realizar estudios longitudinales y comparar la sintomatología e impacto psicológico durante distintas etapas de la pandemia, ya que en el caso de ésta investigación se recolectaron los datos durante el segundo y tercer mes del estado de excepción, donde probablemente los cambios que se llevaron a cabo en aquel momento pudieron condicionar el estado emocional de los participantes. Asimismo, considerar otros factores protectores y su efecto positivo sobre la salud mental de las personas e incluirlos en la elaboración de líneas de intervención que contribuyan al bienestar de la comunidad.

\section{Conflictos de intereses}

Los autores declaran no tener conflictos de intereses

\section{Referencias}

Acosta-Rodríguez, F., Rivera-Martínez, M. y Pulido-Rull, M.A. (2011). Depresión y ansiedad en una muestra de individuos mexicanos desempleados. Journal of Behavior, Health \& Social Issues, 3(1), 35-42. https://doi.org/10.5460/jbhsi. v3.1.27682.

Antúnez, Z. y Vinet, E. (2012). Escalas de depresión, ansiedad y estrés (DASS-21): Validación de la versión abreviada en estudiantes universitarios chilenos. Revista Terapia Psicológica, 30(3), 49-55. http://dx.doi.org/10.4067/S0718-48082012000300005

Antúnez, Z. y Vinet, E. (2013). Problemas de salud mental en estudiantes de una universidad regional chilena. Revista Médica de Chile, 141(2), 209-216. http://dx.doi.org/10.4067/ S0034-98872013000200010

Anguita, V. y Dagnino, P. (2020). Impacto psicológico del aislamiento social. Psicología Hoy, 36. Recuperado de https:// www.uahurtado.cl/wp-images/uploads/2020/06/ Psicolog\%C3\%ADa-Hoy-36.pdf

Asensio-Martínez, A., Magallón-Botaya, R. y García-Campayo, J. (2017). Resiliencia: impacto positivo en la salud física y mental. International Journal of Developmental and Educational Psychology, 2(2), 231-242. https://doi.org/10.17060/ijodaep.2017. n2.v2.1096

Asociación de Municipalidades de Chile, AMUCH (2020). Encuesta Nacional de Salud Mental de las personas en tiempos de pandemia del COVID-19. Recuperado de https:// www.amuch.cl/wp-content/uploads/2020/05/Encuestanacional-de-salud-mental-de-las-personas-en-tiempos-depandemia-del-covid-19-2020.pdf

Baader, T., Rojas, C., Molina, J., Gotelli, M., Alamo, C., Fierro, C., Venezian, S. y Dittus, P. (2014). Diagnóstico de la prevalencia de trastornos de la salud mental en estudiantes universitarios y los factores de riesgo emocionales asociados. Revista Chilena de Neuro-psiquiatría, 52(3), 167-176. http:// dx.doi.org/10.4067/S0717-92272014000300004

Barrera-Herrera, A., Neira-Cofré, M., Raipán-Gómez, P., Riquelme-Lobos, P. y Escobar, B. (2019). Apoyo social percibido y factores sociodemográficos en relación con los síntomas de ansiedad, depresión y estrés en universitarios chilenos. Revista de Psicopatología y Psicología Clínica, 24, 103-123. https://doi.org/10.5944/rppc.23676

Bento, M., Dias, L., Braga, L., Lessa, C., Martinez, F., Rosa, P., \& Azevedo, R. (2006). Spiritual well-being and minor psychiatric disorders in psychology students: a cross-sectional study. Revista de Psiquiatria do Rio Grande do Sul, 28(1), 1-14. http://dx.doi.org/10.1590/S0101-81082006000100003

Brooks, S., Webster, R., Smith, L., Woodland, L., Wessely, S., Greenberg, N., \& Rubin, G. (2020). The psychological impact of quarantine and how to reduce it: rapid review of the evidence. Lancet, 395, 912-920.https://doi.org/10.1016/ S0140-6736(20)30460-8

Cabanyes, J. (2010). Resiliencia: una aproximación al concepto. Revista de Psiquiatría y Salud Mental, 3(4), 145-151. https:// doi.org/10.1016/j.rpsm.2010.09.003

Cáceres, K. (2020). Educación virtual: creando espacios afectivos de convivencia y aprendizaje en tiempos de COVID-19. CienciAmérica, 9(2), 38-44. https://dx.doi.org/10.33210/ca. v9i2.284

Campbell-Sills, L. \& Stein, M. (2007). Psychometric analysis and refinement of the Connor-Davidson resilience scale (CD-RISC): Validation of a 10-item measure of resilience. Journal of Traumatic Stress, 20(6), 1019-1028. https://doi. org/10.1002/jts.20271

Campos, M., Páez, D., Fernandez-Berrocal, P., Igartúa, J.J., Méndez, D., Moscoso, S., Palomero, C., Pérez, J. A., Rodríguez, M., Salgado-Velo, J. y Tasado, C. (2004). Las actividades religiosas como formas de afrontamiento de hechos estresantes y traumáticos con referencia a las manifestaciones del 11-M. Ansiedad y Estrés, 10(2-3), 287-298.

Catalano, D., Chan, F., Wilson, L., Chiu, C.Y., \& Muller, V.R. (2011). The buffering effect of resilience on depression among individuals with spinal cord injury: a structural equation model. Rehabilitation Psychology, 56(3), 200-211. https://doi. org/10.1037/a0024571

Comisión Económica para América Latina y el Caribe, CEPAL (2020). La pandemia del COVID-19 profundiza la crisis de los cuidados en América Latina y el Caribe. Recuperado de https://repositorio.cepal.org/bitstream/handle/11362/45335/5/ S2000261_es.pdf

Connor, K. \& Davidson, J. (2003). Development of a new resilience scale: The Connor-Davidson Resilience Scale (CD-RISC). Depression and Anxiety, 18, 76-82. https://doi. org/10.1002/da.10113

Cova, F. y Rincón, P. (2010). El terremoto y tsunami del 27-F y sus efectos en la salud mental. Terapia Psicológica, 28(2), 179-185. http://dx.doi.org/10.4067/S0718-48082010000200006.

Díaz, C., Quintana, G. y Vogel, E. (2012). Síntomas de depresión, ansiedad y estrés post-traumático en adolescentes siete meses después del terremoto del 27 de febrero de 2010 en Chile. Terapia Psicológica, 30(1), 37-43. http://dx.doi.org/10.4067/ S0718-48082012000100004

Figueroa, R. y Cortés, P. (2016). Propuesta para abordar los problemas de salud mental detectados en el proceso diagnóstico de la Delegación Presidencial para la reconstrucción tras el terremoto del 27 de febrero de 2010. Revista Médica de Chile, 144, 247-252. http://dx.doi. org/10.4067/S0034-98872016000200014 
Garcés, M. y Frías, P. (2020). Corona virus, trabajo y desempleo: los otros efectos de la pandemia. Psicología Hoy, 36. Recuperado de https://www.uahurtado.cl/wp-images/uploads/2020/06/ Psicolog\%C3\%ADa-Hoy-36.pdf

García-Cid, A., Hombrados-Mendieta, I., Gómez-Jacinto, L., Palma-García, M.O. y Millán-Franco, M. (2017). Apoyo social, resiliencia y región de origen en la salud mental y la satisfacción vital de los inmigrantes. Universitas Psychologica, 16(5), 1-14. https://doi.org/10.11144/Javeriana.upsy16-5.asrr

García del Castillo, J., García del Castillo-López, Á., LópezSánchez, C. y Dias, P. (2016). Conceptualización teórica de la resiliencia psicosocial y su relación con la salud. Health and Addictions, 16(1), 59-68. http://www.redalyc.org/articulo. oa? id $=83943611006$

Groome, D. \& Soureti, A. (2004). Post-traumatic stress disorder and anxiety symptoms in children exposed to the 1999 Greek earthquake. British Journal of Psychology, 95(3), 387-397. https://doi.org/10.1348/0007126041528149

Ho, C., Chee, C., \& Ho, R. (2020). Mental health strategies to combat the psychological impact of COVID-19 beyond paranoia and panic. Annals, Academy of Medicine, Singapore, 49(3), 155-160. https://doi.org/10.47102/annals-acadmedsg.202043

$\mathrm{Hu}, \mathrm{T}$., Zhang, D., \& Wang, J. (2015). A meta-analysis of the trait resilience and mental health. Personality and Individual Differences, 76, 18-27.https://doi.org/10.1016/j.paid.2014.11.039

Huarcaya-Victoria, J. (2020). Consideraciones sobre la salud mental en la pandemia de COVID-19. Revista Peruana de Medicina Experimental y Salud Pública, 37(2), 327-334. https://doi.org/10.17843/rpmesp.2020.372.5419

Instituto de Investigación en Ciencias del Comportamiento, IICC (2020). Efectos psicológicos y sociales durante la cuarentena en Bolivia: resultados preliminares. Recuperado de http:// www.iisec.ucb.edu.bo/assets_iisec/publicacion_observatorio/ infografia_final_1.pdf

Ipsos (2020). Coronavirus en Chile. Medición 5. Recuperado de https://www.ipsos.com/sites/default/files/ct/news/ documents/2020-05/tracking_ipsos_covid_19_-_5.pdf

Kar, N., Mohapatra, P., Nayak, K., Pattanaik, P., Swain, S., \&Kar, H. (2007). Post-traumatic stress disorder in children and adolescents one year after a super-cyclone in Orissa, India: Exploring cross-cultural validity and vulnerability factors. $B M C$ Psychiatry, 7(8), 1-9. https://doi.org/10.1186/1471-244X-7-8

Kemper, K., Mo, X., \& Khayat, R. (2015). Are mindfulness and self-compassion associated with sleep and resilience in health professionals? The Journal of Alternative and Complementary Medicine, 21(8), 496-503.https://doi.org/10.1089/acm.2014.0281

Korc, M., Hubbard, S., Suzuki, T. y Jimba, M. (2016). Salud, resiliencia y seguridad humana: hacia la salud para todos. Recuperadodehttps://iris.paho.org/bitstream/handle/10665.2/28305/ 9784889071481_spa.pdf? sequence $=1 \&$ isAllowed $=\mathrm{y}$

Lai, J., Ma, S., Wang, Y., Cai, Z., Hu, J., Wei, N., Wu, J., Du, H., Chen, T., Li, R., Tan, H., Kang, L., Yao, L., Huang, M., Wang, H., Wang, G., Liu, Z., \&Hu, S. (2020). Factors Associated with Mental Health Outcomes Among Health Care Workers Exposed to Coronavirus Disease 2019. JAMA Network Open, 3(3), 1-12. https://doi.org/10.1001/ jamanetworkopen.2020.3976

Lazarus, R.S. \& Folkman, S. (1984). Stress, appraisal, and coping. Springer Publishing Company.
López, E., Guevara, V. y Quinteros, D. (2016). Religiosidad y resiliencia en estudiantes de psicología de una universidad privada de Lima Este. Revista Cientifica de Ciencias de la Salud, 9(2), 26-31. https://doi.org/10.17162/rccs.v9i2.650

Lovibond, S.H. \& Lovibond, P.F. (1995). Manual for the depression anxiety stress scales. Psychology Foundation of Australia.

Lozano-Díaz, A., Fernández-Prados, J., Figueredo, V. y Martínez, A.M. (2020). Impactos del confinamiento por el COVID-19 entre universitarios: Satisfacción Vital, Resiliencia y Capital Social Online. International Journal of Sociology of Education, 79-104. http://dx.doi.org/10.17583/rise.2020.5925

Mazza, C., Ricci, E., Biondi, S., Colasanti, M., Ferracuti, S., Napoli, C., \& Roma, P. (2020). A nationwide survey of psychological distress among italian people during the COVID-19 pandemic: Immediate psychological responses and associated factors. International Journal of Environmental Research and Public Health, 17, 3165. https://doi.org/10.3390/ ijerph17093165

Ministerio del Interior y Seguridad Pública (2020). Declara Estado de Excepción Constitucional de catástrofe, por calamidad pública, en el territorio de Chile. Diario oficial de la República de Chile, 104. Recuperado de https://www.diariooficial.interior. gob.cl/publicaciones/2020/03/18/42607-B/01/1742691.pdf

Mofidi, M., DeVellis, R., DeVellis, B., Blazer, D., Panter, A., \& Jordan, J. (2007). The relationship between spirituality and depressive symptoms: Testing psychosocial mechanisms. The Journal of nervous and mental disease, 195(8), 681-688. https://doi.org/10.1097/NMD.0b013e31811f4038

Moorhouse, A. \& Caltabiano, M. (2007). Resilience and unemployment: Exploring risk and protective influences for the outcome variables of depression and assertive job searching. Journal of Employment Counseling, 44, 115-125. https://doi.org/10.1002/j.2161-1920.2007.tb00030.x

Moreno, G. (2020). Pensamientos distorsionados y ansiedad generalizada en COVID-19. CienciAmérica, 9(2), 251-255. http://dx.doi.org/10.33210/ca.v9i2.314

Notario-Pacheco, B., Solera-Martínez, M., Serrano-Parra, M., Bartolomé-Gutiérrez, R., García-Campayo, J., \& MartínezVizcaíno, V. (2011). Reliability and validity of the Spanish version of the 10-item Connor-Davidson Resilience Scale (10item CD-RISC) in young adults. Health and Quality of Life Outcomes, 9(63), 1-6. https://doi.org/10.1186/1477-7525-9-63

Orellana, C. y Orellana, L. (2020). Predictores de síntomas emocionales durante la cuarentena domiciliar por pandemia de COVID-19 en El Salvador. Actualidades en Psicología, 34(128), 103-120. https://doi.org/10.15517/ap.v34i128.41431

Organización Mundial de la Salud, OMS (2020a). Brote de enfermedad por coronavirus (COVID-19). Recuperado de https://www. who.int/es/emergencies/diseases/novelcoronavirus-2019

Organización Mundial de la Salud, OMS (2020b). WHO, situation report-51. Recuperado de https://www.who.int/docs/defaultsource/coronaviruse/situationreports/20200311-sitrep-51covid-19.pdf?sfvrsn=1ba62e57_10

Organización Mundial de la Salud, OMS (2020c). Mental health and psychosocial considerations during the COVID-19 outbreak. Recuperado de https:/www.who.int/publications/i/ item/mental-health-and-psychosocial-considerations-duringthe-covid-19-outbreak 
Organización Panamericana de la Salud, OPS, y Organización Mundial de la Salud, OMS (1997). Estado de Arte en Resiliencia. Recuperado de https:/www.paho.org/Spanish/ HPP/HPF/ADOL/Resil6x9.pdf

Ozamiz-Etxebarria, N., Dosil-Santamaria, M., Picaza-Gorrochategui, M. y Idoiaga-Mondragon, N. (2020). Niveles de estrés, ansiedad y depresión en la primera fase del brote del COVID-19 en una muestra recogida en el norte de España. Cadernos de Saúde Pública,36(4), 1-10.https://doi.org/10.1590/0102-311x00054020.

Pidgeon, A., Rowe, N., Stapleton, P., Magyar, H., \& Lo, B. (2014). Examining characteristics of resilience among university students: an international study. Open Journal of Social Sciences, 2, 14-22. http://dx.doi.org/10.4236/jss.2014.211003

Román, F., Santibáñez, P. y Vinet, E. (2016). Uso de las Escalas de Depresión Ansiedad Estrés (DASS-21) como instrumento de tamizaje en jóvenes con problemas clínicos. Acta de Investigación Psicológica, 6(1), 2325-2336. https://doi. org/10.1016/S2007-4719(16)30053-9

Salgado, A. (2014). Revisión de estudios empíricos sobre el impacto de la religión, religiosidad y espiritualidad como factores protectores. Propósitos y Representaciones, 2(1), 121-159. http://dx.doi.org/10.20511/pyr2014.v2n1.55

Sandín, B., Chorot, P., García-Escalera, J., y Valiente, R. M. (2021). Impacto emocional de la pandemia de COVID-19 durante el periodo de confinamiento en España: Factores protectores y factores de riesgo/vulnerabilidad. Acción Psicológica, 18, 27-44. https://doi.org/10.5944/ap.18.1.29520

Sandín, B., Valiente, R.M., García-Escalera, J. y Chorot, P. (2020). Impacto psicológico de la pandemia de COVID-19: Efectos negativos y positivos en población española asociados al periodo de confinamiento nacional. Revista de Psicopatología y Psicología Clínica, 25(1), 1-22. https://doi.org/10.5944/rppc.27569

Seok, J.H., Lee, K.U., Kim, W., Lee, S. H., Kang, E. H., Ham, B. J., Yang, J. C., \& Chae, J.H. (2012). Impact of early-life stress and resilience on patients with major depressive disorder. Yonsei Medical Journal, 53(6), 1093-1098. https://doi. org/10.3349/ymj.2012.53.6.1093

Shevlin, M., McBride, O., Murphy, J., Miller, J., Hartman, T., Levita, L., Mason, L., Martinez, A. P., McKay, R., Stocks, T., Bennett, K. M., Hyland, P., Karatzias, T., \&Bentall, R. (2020). Anxiety, Depression, Traumatic Stress, and COVID-19 Related Anxiety in the UK General Population During the COVID-19 Pandemic. In preparation. https://doi.org/10.31234/osf.io/hb6nq

Sinding, J. (2020). In crisis, we pray: Religiosity and the Covid-19 pandemic. Covid Economics, 20, 52-108.

Ticehurst, S., Webster, R., Carr, V., \& Lewin, T. (1996). The psychosocial impact of an earthquake on the elderly. International Journal of Geriatric Psychiatry, 11, 943-951. https://doi.
org/10.1002/(SICI)1099-1166(199611)11:11<943:AIDGPS412>3.0.CO;2-B

Tolin, D. \& Foa, E. (2006). Sex differences in trauma and posttraumatic stress disorder: A quantitative review of 25 years of research. Psychological Bulletin, 132(6), 959-992. https://scite.ai/reports/10.1037/0033-2909.132.6.959

Tomyn, A. \& Weinberg, M. (2016). Resilience and subjective wellbeing: A psychometric evaluation in young australian adults. Australian Psychologist, 53(1), 68-76. https://doi. org/10.1111/ap.12251

Ubillos, S., González, J.L., Puente, A. y Gracia, M. (2020). Afrontando el impacto del COVID-19: resultados preliminares III. Recuperado de https://riubu.ubu.es/bitstream/ handle/10259/5360/Afrontando_el_impacto_de1\%20 COVID19-3.pdf? sequence $=1$

United Nations Children's Fund, UNICEF (2019). Risk-informed education programming for resilience guidance note. Recuperado de https://www.unicef.org/media/65436/file/ Risk-informed $\% 20$ education $\% 20$ programming $\% 20$ for $\% 20$ resilience:\%20Guidance\%20note.pdf

Vizoso, C. (2019). Resiliencia, optimismo y estrategias de afrontamiento en estudiantes de Ciencias de la Educación. Psychology, Society, \& Education, 11(3), 367-377. http://dx. doi.org/10.25115/psye.v11i3.2280

Wanderley, F., Losantos, M., Tito, C. y Arias, A.M. (2020). Los impactos sociales y psicológicos del COVID-19 en Bolivia. Serie Reflexiones sobre la Pandemia en Bolivia, 3. Recuperado de http://repositorio.ucb.edu.bo/xmlui/bitstream/handle/UCB/247/ iisec_3.pdf?sequence $=1$

Wang, C., Pan, R., Wan, X., Tan, Y., Xu, L., Ho, C., \& Ho, R. (2020). Immediate Psychological Responses and Associated Factors during the Initial Stage of the 2019 Coronavirus Disease (COVID-19) Epidemic among the General Population in China. International Journal of Environmental Research and Public Health, 17, 1729. https://doi.org/10.3390/ijerph17051729

Wenham, C., Smith, J., \& Morgan, R. (2020). COVID-19: the gendered impacts of the outbreak. The Lancet, 395(10227), 846-848. https://doi.org/10.1016/S0140-6736(20)30526-2

Xiao, C. (2020). A novel approach of consultation on 2019 novel coronavirus (COVID-19)-related psychological and mental problems: structured letter therapy. Psychiatry Investigation, 17(2), 175-176. https://doi.org/10.30773/pi.2020.0047

Yu, X.N., Lau, J.T.F., Mak, W.W.S., Cheng, Y.M., Lv, Y.H., \& Zhang, J.X. (2009). Risk and protective factors in association with mental health problems among people living with HIV who were former plasma/blood donors in rural China. AIDS care: Psychological and Socio-medical Aspects of AIDS/HIV, 21(5), 645-654. https://doi.org/10.1080/09540120802459770 